\title{
Design and Dynamic Performance Research of Underwater Inspection Robots
}

\author{
Guojun Chen $\left(\mathbb{D},{ }^{1}\right.$ Wei Chen, ${ }^{1}$ Zhiming Wang, ${ }^{2}$ Tiezheng Guo, ${ }^{1}$ Ximing Xia $\mathbb{D},{ }^{1}$ \\ and Lijun $\mathrm{Xu} \mathbb{D}^{1}$ \\ ${ }^{1}$ Nanjing Institute of Technology, Nanjing, Jiangsu, China \\ ${ }^{2}$ Key Laboratory of Crop Harvesting Equipment Technology of Zhejiang Province, Zhejiang, China \\ Correspondence should be addressed to Guojun Chen; chengj@njit.edu.cn
}

Received 18 January 2022; Revised 6 February 2022; Accepted 7 February 2022; Published 24 February 2022

Academic Editor: Xin Ning

Copyright (C) 2022 Guojun Chen et al. This is an open access article distributed under the Creative Commons Attribution License, which permits unrestricted use, distribution, and reproduction in any medium, provided the original work is properly cited.

With the construction of a large number of power plants along the river and the coast, more and more power plants have adopted open water intake in the circulating water pool of the power plant and the number of underwater operations is increasing. In the detection of complex underwater environments, underwater robots have been widely used as a kind of underwater intelligent equipment. However, the current underwater robots generally have problems such as weak endurance, low intelligence, poor detection ability, and large environmental disturbance. The underwater robot designed in this paper is able to accomplish various rotational and flat movement works in the water and also can realize the monitoring of the water environment. In this paper, the underwater dynamic performance of the underwater robot is studied, the coordinate system and motion equation are established, the hydrodynamic analysis is carried out, and the dynamic characteristics of the underwater robot are tested. The experimental results show that the underwater robot has achieved good results in underwater motion control and its dynamic performance, showing the advantages of good robustness and accuracy, high control accuracy, and good dynamic characteristics.

\section{Introduction}

The circulating cooling water of the condensers of thermal power plants in coastal areas mostly uses seawater, and the circulating water pool is used as the water intake for the cooling water of the condensers of the power plants $[1,2]$. Due to the complex marine environment, there are a series of production difficulties $[3,4]$. On the one hand, there are many marine organisms. If the water intake of the cold source of the power plant is invaded, the filter screen will be blocked, and the condenser will lose a lot of cooling water. Once the cooling source of the power plant is lost, it will directly lead to the unplanned shutdown of the unit. In recent years, there have been many serious incidents of marine organisms attacking the cold source water intake of the power plant in coastal power plants in China. On the other hand, when the circulating water pump is repaired, it is necessary to lift the large gate to isolate the circulating water pump, but because the inlet gate cannot be closed tightly, the isolation is not in place or cannot be effectively isolated $[5,6]$. Due to the limitation of the location, it is difficult to effectively block the leakage of the inlet gate manually $[7,8]$.

Underwater robots have become an indispensable tool for human exploration and development of the ocean and are widely used in ocean development, underwater exploration, underwater operations, underwater rescue, and other fields [9-11]. Underwater robots can perceive the environment, identify underwater objects, plan paths, avoid obstacles autonomously, and complete underwater tasks $[12,13]$. The application of underwater robots in the complex underwater environment of circulating pools in power plants can effectively solve difficult problems such as underwater monitoring, maintenance, cleaning, and construction of circulating pools [14-16].

This paper focuses on the production problems such as the difficulty of artificially plugging the leakage of the inlet gate of the circulating pump room of the coastal power plant, the difficulty and high risk of manual removal of debris in 
the trash rack at the water intake, and the lack of timely artificial monitoring of marine life in the water intake, which leads to the interruption of circulating water. An underwater inspection robot for a circulating pool is developed to monitor the underwater environment in real time and maintain and repair the underwater equipment of the circulating pool. The underwater movement and operation of the underwater robot are based on the underwater dynamics model of the underwater robot. In order to establish an accurate dynamic model, the main disturbance factors of the underwater robot are deeply analyzed in this paper, and the dynamic characteristics of the underwater robot are also studied.

\section{Underwater Robot System Design}

2.1. Underwater Robot System Components. The underwater robot system designed in this paper can be divided into three major components: the control system, the observation system, and the carrier system. The overall design of the underwater robot is shown in Figure 1.

The control system is a system that processes and analyzes external information and can sense the underwater environment through sensors [17-19]. The underwater robot not only receives control commands in real time but also feeds back relevant underwater information in real time $[20,21]$. The underwater robot designed in this paper is a cabled remote control type, which can realize the functions of advancing, retreating, turning, observing, and underwater grasping in the water.

The observation system includes an underwater camera, a depth gauge, and a robotic arm, which can transmit the state of the underwater robot and the information of the underwater environment to the man-machine system of the ground station on the shore through the cable. The operator controls the underwater robot and the mechanical arm to complete the required operations.

The carrier system includes the shape structure of the underwater robot, the sealed cabin, the floating body, the propeller, the steering gear, and other hardware equipment [22-24]. Among them, the sealed cabin is used to store the electronic components of the underwater robot, including the main control board, sensors, and other electrical components. The floating body is to provide buoyancy to the underwater robot. The propeller and the steering gear belong to the power system. By controlling the speed and steering of the motor, the propeller realizes the rotation and translation of the fuselage, and the steering gear is mainly used for the attitude adjustment of the underwater robot. The technical parameters of the underwater robot are shown in Table 1.

\subsection{Mechanical Structure Design of Underwater Robots}

\subsubsection{Underwater Robot Body Structure and Architectural Materials}

(1) Overall Shape Design of Underwater Robots. Due to the different purposes and working methods, the shape and structure of underwater robots are very different.
Considering all aspects, the underwater robot designed in this paper adopts the frame shape structure, which can ensure navigation performance and reduce the resistance in the water as much as possible during the navigation. At the same time, the frame-shaped underwater robot can carry more equipment, which is conducive to maximizing the use of space, and it is convenient to adjust the center, buoyancy, and resistance of the whole machine, as well as the assembly and debugging of the underwater robot.

(2) Architectural Material Selection.. In the selection of the overall architecture materials, after analyzing the advantages and disadvantages of engineering plastics, aluminum alloys, titanium alloys, and stainless steel and considering the actual engineering complexity and the strength, stiffness, designability, manufacturability, economy, and corrosion resistance of the materials, the stainless steel alloy material was used for the underwater robot shape architecture designed in this paper. The stainless steel alloy material can effectively avoid corrosion when the underwater robot works underwater for a long time, while the stiffness and strength of the stainless steel alloy material can effectively play a protective role. Of course, from these two underwater bionic robots, we can also see that there are two huge markets for the application of underwater robots at present: the education industry and the fishing and animal husbandry industry. A highly integrated, low-cost underwater bionic fish needs to be designed urgently.

\subsubsection{Shape and Material Selection of the Closed Cabin}

(1) Shape Selection of the Closed Cabin. Because underwater robots work underwater, they must use closed pressure chambers to install electronic equipment to ensure that there will be no leakage damage due to water pressure and corrosion. Therefore, the cabin of the underwater robot should have sufficient strength and reliable sealing. Underwater robots have various cabin shapes. Considering that the cylindrical cabin can effectively utilize the internal space of the cabin and facilitate the placement of electronic components, the cylindrical cabin is chosen as the sealed cabin in this paper.

(2) Material Selection for the Closed Cabin. The working environment of the underwater robot puts forward many special requirements for the material of the cabin. Corrosion resistance, strength, impact toughness, economy, etc. must be considered when selecting the material of the cabin. In addition, as part of providing buoyancy, the density of the cabin material should not be too high. Through the selection and consideration of various materials, acrylic plastic is finally selected as the cabin.

(3) Sealing of the Cabin. After the underwater robot has completed its underwater operations, it often needs to be serviced, so the pressure-resistant compartment body must have a removable head to allow for the assembly and removal of the electronics inside the housing. A good seal should be ensured between the detachable head and the cabin so that the underwater robot does not leak, and the components and 


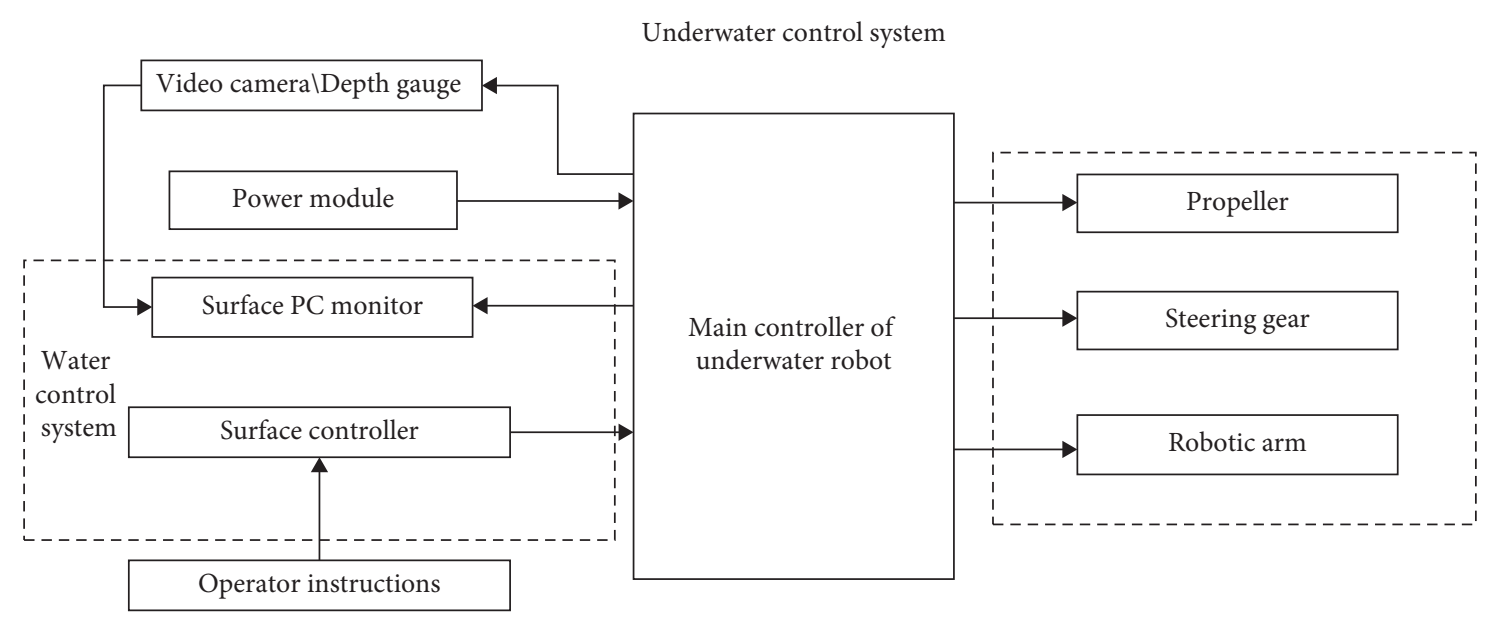

Figure 1: Underwater robot general design diagram.

TABle 1: Technical parameters of the underwater robot.

\begin{tabular}{lc}
\hline Technical indicators & Parameter \\
\hline Speed & $0.5 \sim 1 \mathrm{~m} / \mathrm{s}$ \\
Size & $0.45 \mathrm{~m} \times 0.45 \mathrm{~m} \times 0.2 \mathrm{~m}$ \\
Diving depth & $5 \mathrm{~m}$ \\
Weight & Approximately equal to $8 \mathrm{~kg}$ \\
\hline
\end{tabular}

instruments in the pressure-resistant shell are not damaged. In order to facilitate the assembly and disassembly of the detachable head and ensure reliable sealing, a rubber O-ring is used as the sealing element in this paper.

2.2.3. Floating Body Module Structure Design. This paper designs a cabled remote-controlled, floating underwater robot. Therefore, in order to achieve the underwater suspension control of the underwater robot with a small propulsion force, and in the case of a power failure, the robot can automatically float up and can be realized on the water surface. To float autonomously to facilitate observation and recovery by shore operators, it is necessary to provide sufficient buoyancy for underwater robots. The cabin of the underwater robot will generate some buoyancy, but its own buoyancy is far less than the gravity of the entire robot. Therefore, it is necessary to arrange solid buoyancy blocks on the underwater robot to provide most of the buoyancy for the entire underwater robot, so as to ensure that the underwater robot can sail in a suspended state in the water.

In order to ensure that the robot can float on the water, according to the principle that the overall gravity to buoyancy ratio of the underwater robot is designed to be 1 : 1.1 , the floating body module needs to provide $65 \mathrm{~N}$ of buoyancy according to the calculation. In order to ensure the stability of the underwater robot's movement in the water, the floating body module needs to be designed into a symmetrical structure. Combined with the above-mentioned underwater robot body structure, this paper adopts 8 buoyancy modules, which are symmetrically placed on the underwater robot. The buoyancy module is made of foam XPS specially used for mechanical architectural design models. XPS foam is high-density foam with high compressive strength and extremely low water absorption. Its parameters are shown in Table 2. The floating body module is processed by an electrothermal cutting machine, and there are two external dimensions. One is the floating block installed on the four corners of the main body of the underwater robot, and its size is $12 \mathrm{~cm} \times 8 \mathrm{~cm} \times 10 \mathrm{~cm}$, and the other is the floating block installed on the steering gear, and its size is $10 \mathrm{~cm} \times 6 \mathrm{~cm} \times 5 \mathrm{~cm}$.

2.2.4. Propulsion System Design. The propellers used in this paper all have the same performance. The forward power of the propeller is the same as the reverse power. The positional layout of the propellers is related to the motion performance requirements of the involved underwater robots. In order to ensure that the designed underwater robot has the functions of advancing, retreating, turning, and lifting in the water, this paper chooses to install four propellers to realize the above functions.

In summary, the physical map of the underwater robot designed in this paper is shown in Figure 2.

\subsection{Design of the Electronic Control System for Underwater} Robots. The hardware system of the electrical part of the underwater robot can be functionally classified into underwater robot power supply system hardware and underwater robot control system hardware. The control system hardware is divided by location into three main parts: (1) the ground station, including the remote control and computer; (2) the body of the underwater robot with the controller; and (3) the connection cable (the connection cable has a special role, both as a power supply line and a signal transmission line). The hardware system block diagram of its electrical part is shown in Figure 3. The physical map of the ground station, cable, and underwater robot is shown in Figure 4.

2.3.1. Underwater Robot Power Supply System. The underwater robot is connected to the battery on the shore through a cable, and the battery can provide enough energy for the underwater robot to complete the underwater work. 
Table 2: Performance index of XPS foam.

\begin{tabular}{lcccc}
\hline Compressive strength & Density & Water soluble & Water absorption rate & Color \\
\hline $350 \mathrm{kPa}$ & $40 \mathrm{~kg} / \mathrm{m} 3$ & Insoluble in water & $<1 \%$ & Light yellow \\
\hline
\end{tabular}

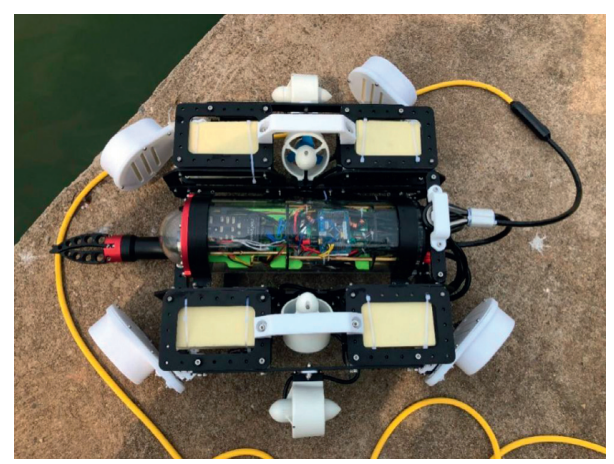

Figure 2: Physical map of an underwater robot.

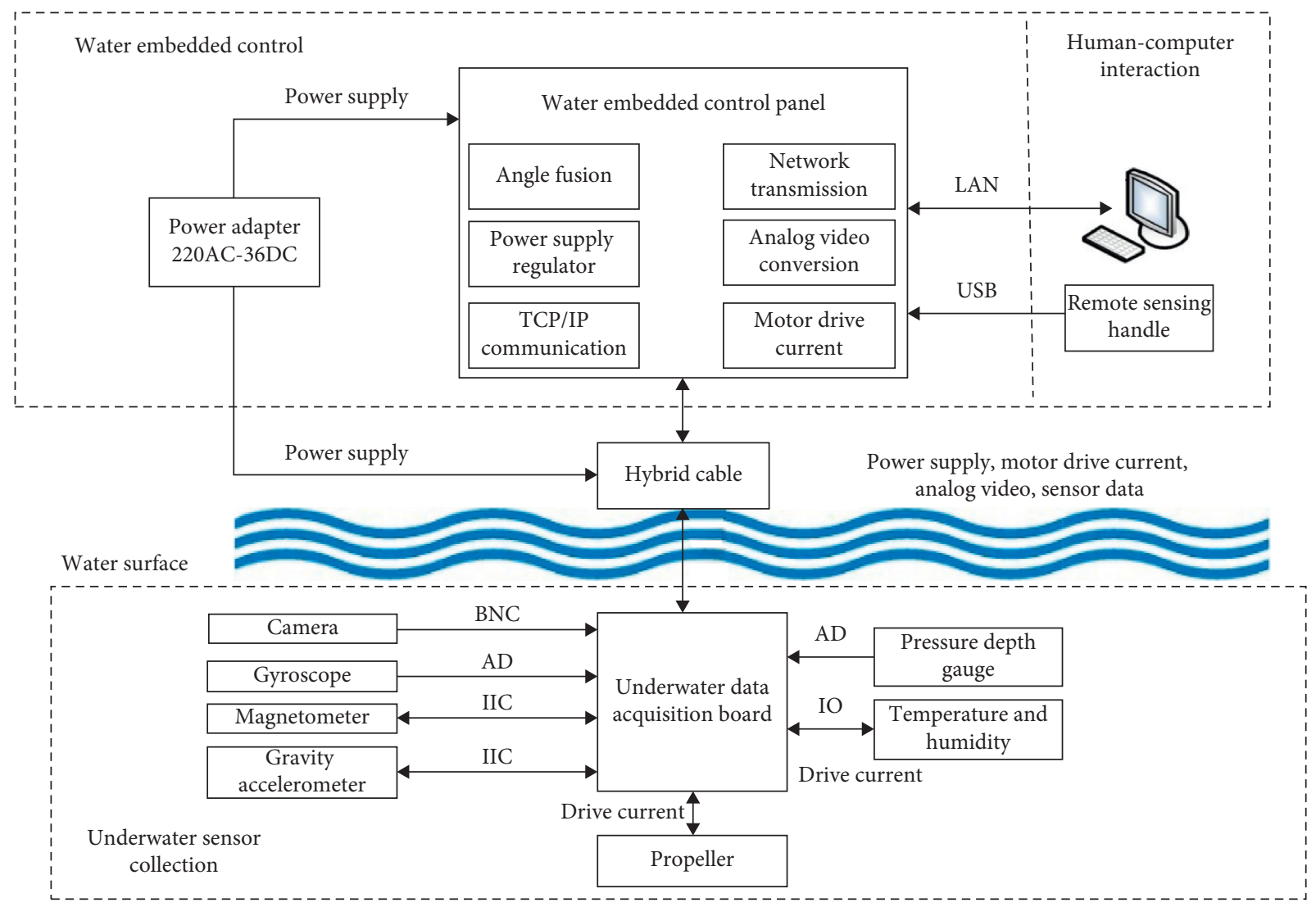

Figure 3: The hardware system block diagram of the electrical part of the underwater robot.

The rated discharge voltage of the shore battery is $48 \mathrm{~V}$, and the electrical components in the underwater robot cabin include control devices such as pixhawk2.4.8 flight control, Raspberry $\mathrm{Pi}$, and stm32 controller, as well as thrusters, steering gears, and mechanical grippers. Different electrical devices have different voltage requirements. If the $48 \mathrm{~V}$ power supply is directly supplied to the devices in the cabin, it will cause damage to the electrical devices. Therefore, it is necessary to use DC-DC to step down the $48 \mathrm{~V}$ voltage differently. Considering the cost and the requirements of the rated working voltage of the device, this product adopts a three-stage step-down circuit, which first reduces the $48 \mathrm{~V}$ to $24 \mathrm{~V}$ and then uses the 24-12VBUCK circuit to reduce the voltage to $12 \mathrm{~V}$ to provide power for the mechanical claw. The third-stage BUCK circuit is then used to reduce the voltage to $6 \mathrm{~V}$ to supply power to the Raspberry $\mathrm{Pi}$ controller, flight control, stm32 controller, and power systems such as thrusters and steering gear. 


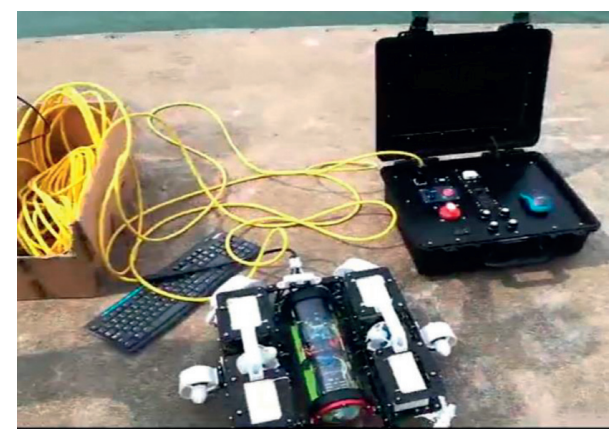

Figure 4: Physical map of the ground station, cable, and underwater robot.

\subsubsection{Control Hardware System}

(1) The Underwater Hardware Control Part of the Underwater Robot. In order to better realize the stable and accurate control of the underwater robot, this paper adopts the opensource system scheme to construct the control system of the underwater robot. This paper adopts the Pixhawk-based flight control and Raspberry Pi open-source system as the control system of this underwater robot. At the same time, in order to improve the accuracy of the control of the underwater steering gear, this paper uses the stm 32 control board and the Raspberry Pi controller to work together to adjust the angle of the steering gear to balance the underwater motion posture of the underwater robot.

(2) Cable. The cable is the connection channel between the underwater robot and the ground station on the shore. Because the transmission attenuation of the WIFI signal in the water is large, the information collected by the underwater robot cannot be effectively uploaded to the ground station. The control instructions issued by the operator at the ground station are also difficult to be received by the underwater robot, and the functions of the underwater robot in deep water cannot be effectively realized, which affects the effect of underwater operations. Therefore, this paper adopts the cable as the channel of information transmission to facilitate the exchange of information between underwater and water.

(3) Ground Station. As a water control system, the ground station consists of electrical hardware: a remote control handle and a man-machine interface. The operator on the shore controls the underwater robot through the computer and the handle, realizes the underwater movement of the underwater robot and the corresponding camera and grasping functions, and understands the underwater information through the man-machine interface:

(1) Remote Control Handle. The operator controls the underwater robot through the remote control handle of the ground station. The command transmission of the ground station and the information transmission of the underwater robot are connected by cables according to the TCP/IP communication protocol. Issue attitude adjustment commands transmit information to the underwater robot through the remote control handle. Control commands transmit information to the capsule's controller via a connected cable. The controller transmits signals to the steering gear, the thruster, and the mechanical arm through the interface to realize the underwater attitude adjustment, movement direction adjustment, and underwater operation of the underwater robot.

(2) Human-Machine Interface. The man-machine interface of the ground station computer is the core of the whole control system. Temperature (1) represents the temperature in the electronic cabin, Temperature (2) represents the temperature outside the electronic cabin; Pilot Gain represents the thruster gear; Voltage represents the current voltage.

\subsection{Software System Design of Underwater Robots}

2.4.1. Software System Design. The purpose is achieved according to the design function of the underwater robot. Under the control of the ground station, the underwater robot needs to complete the basic movements in the water in four directions, the underwater observation of the underwater camera, and the action of the mechanical claw and other practical functions and transmit the underwater information to the ground station to form visual data, images or videos, etc., for the shore operator to analyze and make decisions. Its software control block diagram is shown in Figure 5.

2.4.2. Critical Process Software Flow. This section shows the software flowchart of the key processes as follows, as shown in Figure 6, which is the flowchart of the humancomputer interaction unit designed in this paper. The first step is to link the server, complete the communication between the underwater robot and the computer, and then wait for the keys to be pressed. If the control key is pressed, the control command is sent to the robot to complete the corresponding action in the water and wait for the next control command to come; if the control key is not pressed, it enters the secondary interface while returning to the line graph and waiting for the key to be pressed again. It also waits for the information sent back by the underwater robot sensors, stores the real-time information sent back by the sensors locally, and refreshes the HMI in real time to display the sensor information in the HMI.

Figure 7 shows the flow chart of the underwater robot sensor acquisition unit designed in this paper. Send the settings to the UART0, 1, 2 serial ports through the internal program, set the ADC acquisition and $\mathrm{I} 2 \mathrm{C}$, and complete the initialization. Firstly, I2C is used to collect magnetometer and gravitational acceleration, and ADC is used to collect angular velocity to complete the motion control of the car. At the same time, ADC is used to collect depth gauge and temperature, which is sent to the computer through UART0 to determine whether the data is sent and trigger different interrupts to complete corresponding control. 


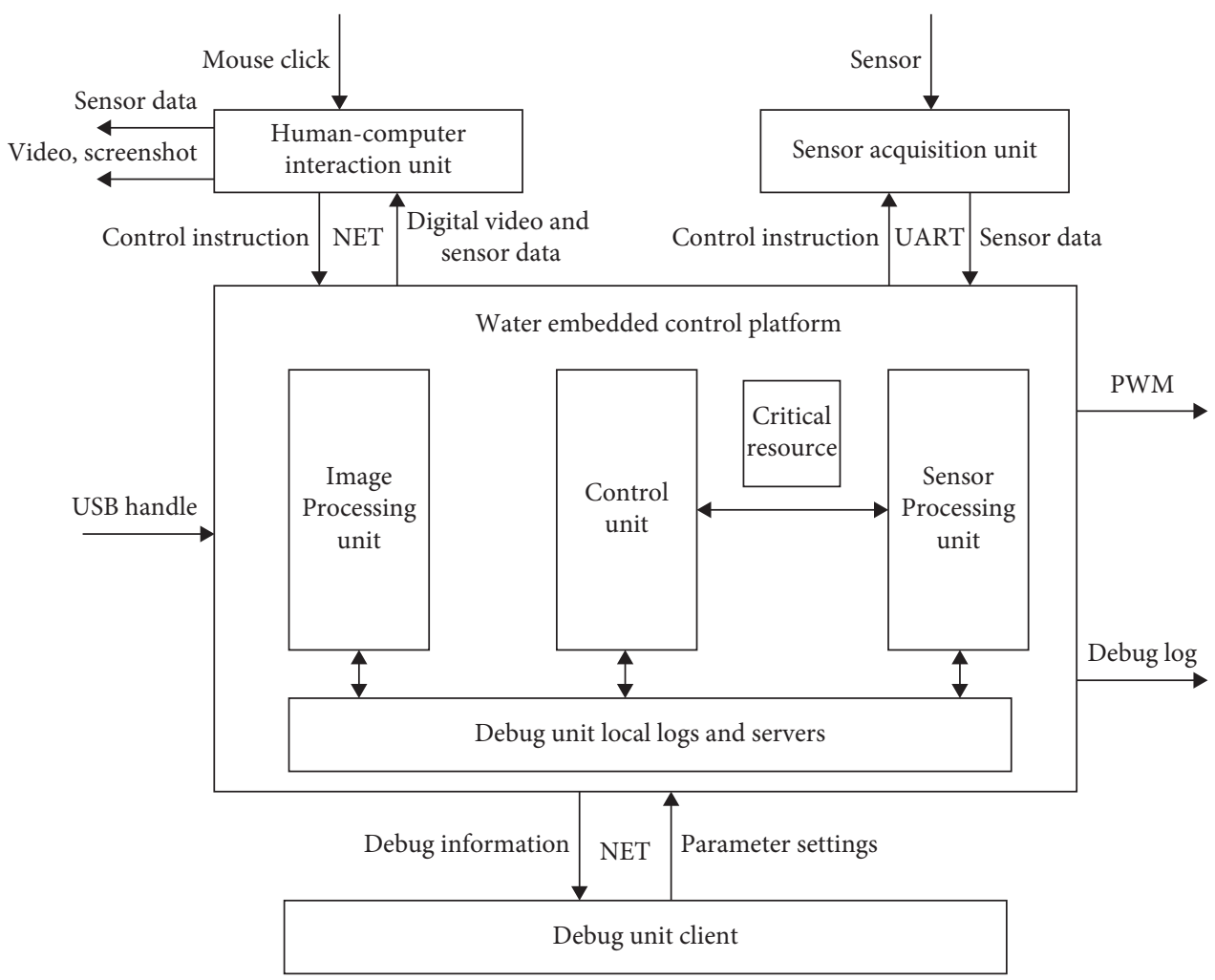

Figure 5: Software system block diagram.

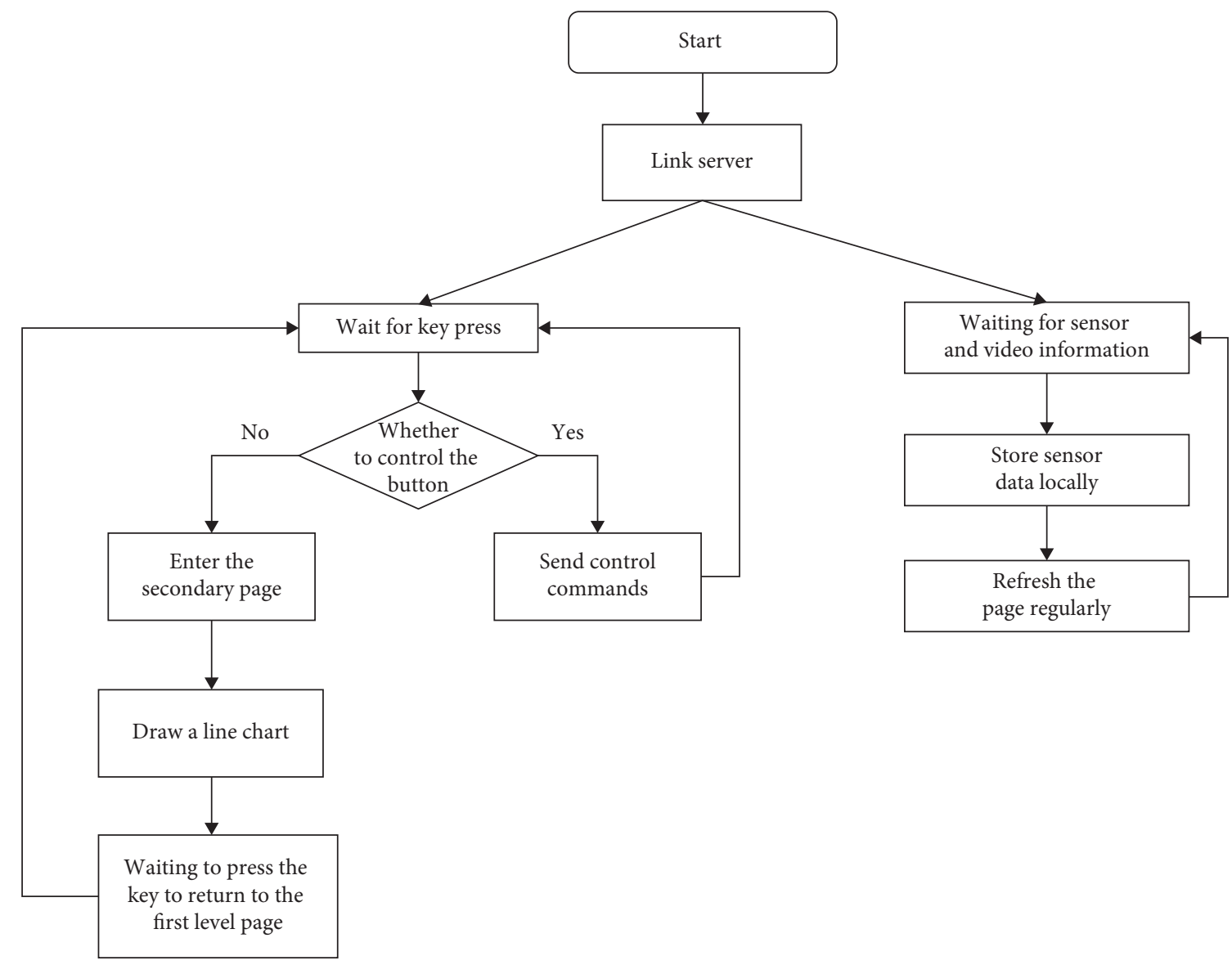

FIgURE 6: Human-computer interaction unit flow chart. 

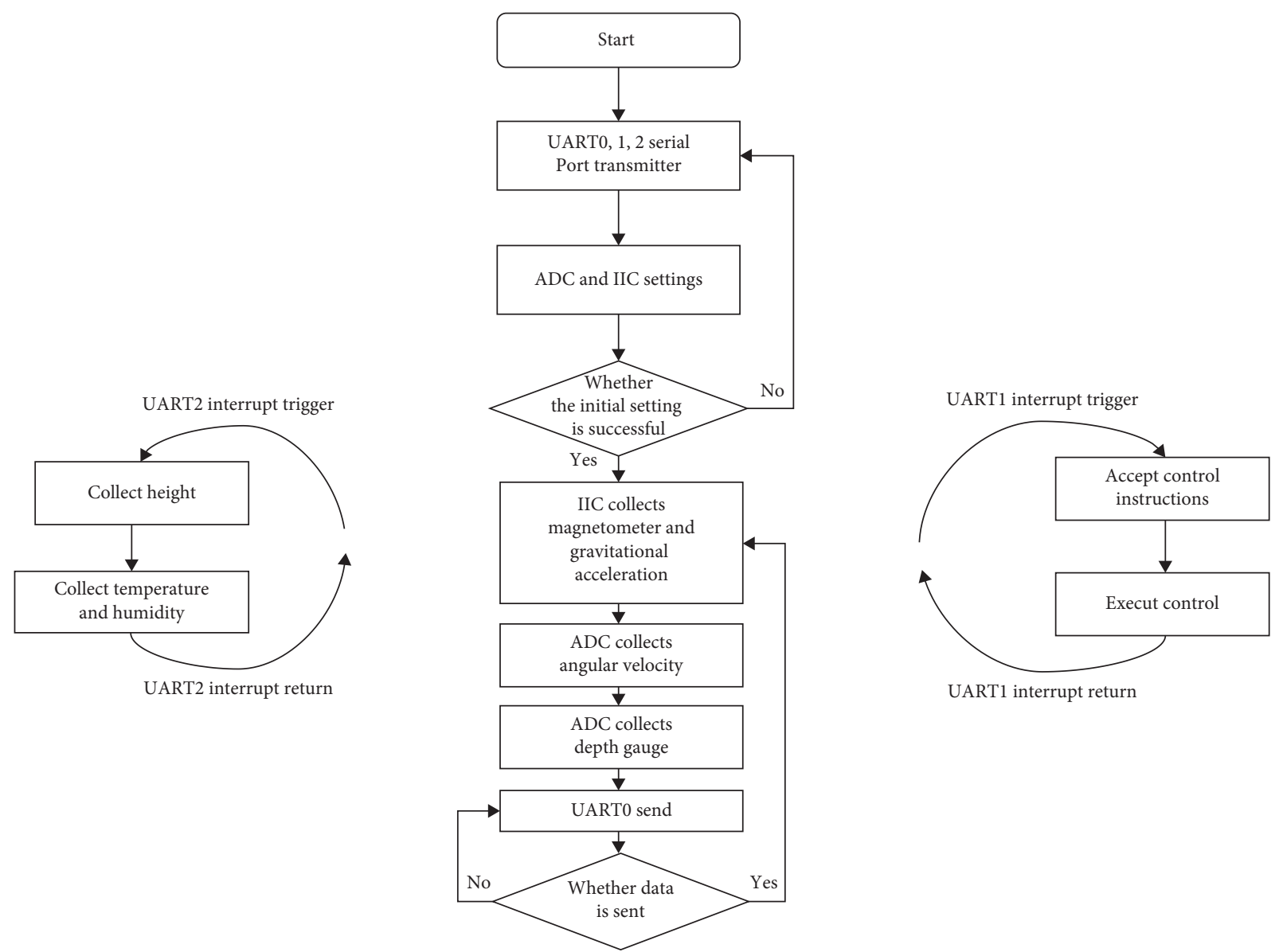

FIgURE 7: Flow chart of the sensor acquisition unit.

\section{Underwater Robot Dynamics Study}

When the underwater robot operates autonomously underwater, it uses the underwater robot as a carrier and is equipped with various sensors and operating equipment. However, due to the complex and changeable underwater environment and the large number of interference sources, the force of the underwater robot is complex, so the dynamic modeling of the underwater robot is more difficult. In this paper, research work has been carried out on the coordinate system of the underwater robot's dynamic system, the equations of the motion system, and the hydrodynamic analysis.

3.1. Establishing the System Coordinate System of the Underwater Robot. Taking the mechanical body of the underwater robot as the research object, the spatial six-degreeof-freedom nonlinear mathematical model of the underwater robot is established according to the momentum theorem under reasonable motion assumptions. In this paper, the underwater robot dynamics modeling method based on the Guttler equation is used to establish the kinematics and dynamics model of the underwater robot. Considering the rigid body dynamics of the underwater robot and the influence of the underwater current on it, two different coordinate systems are used. The first is the inertial coordinate system $E-\xi \eta \zeta$. The coordinate system is fixed with the Earth and is used to describe the motion of the underwater robot in space. The second is the motion coordinate system $O-x y z$. The coordinate system is fixedly connected with the underwater robot and is used to describe the body motion of the underwater robot.

As shown in Figure 8 , the $x$-axis points to the longitudinal axis of the underwater robot, the forward is positive, the $y$-axis points to the starboard, the $z$-axis is determined by the right-hand coordinate system, and the $O$ point coincides with the center of gravity of the underwater robot. In the inertial coordinate system, the origin $E$ is fixed to the Earth, the $E-\xi$ and $E-\eta$ axes are parallel to the horizontal plane, and the $E-\xi$ axis points to the center of the Earth and is perpendicular to the horizontal plane. In the motion coordinate system, the origin $\mathrm{O}$ is fixed to the robot, the $\mathrm{O}-x$ axis points to the forward direction of the underwater robot, the $O-y$ axis points to the starboard side of the underwater robot, and the $\mathrm{O}-z$ axis points to the bottom of the underwater robot.

The underwater robot has six motion postures, and the motion of the underwater robot is a compound motion of various motions. The coordinate origin $O$ is relative to the speed $V$ of the inertial coordinate system, the angular velocity $Q$ around the origin, the force $F$ of the underwater 


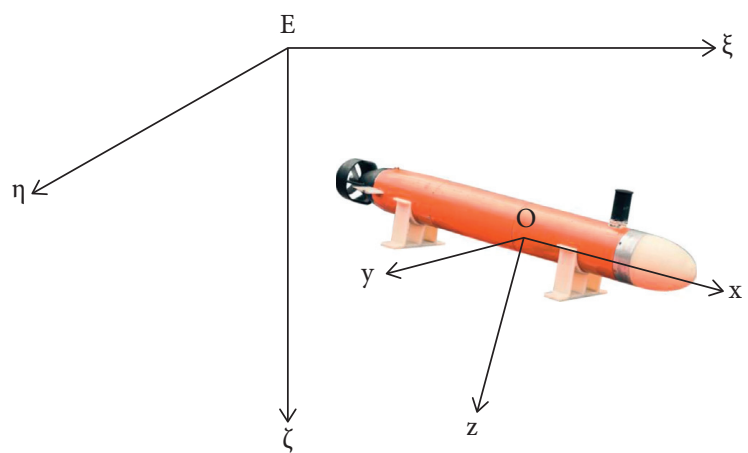

Figure 8: Establishment of the underwater robot coordinate system.

robot, and the torque $M$ of the underwater robot. The inertial coordinate system and the motion coordinate system are transformed by three rotations:

$$
\left[\begin{array}{l}
\xi \\
\eta \\
\zeta
\end{array}\right]=\left[\begin{array}{ccc}
\cos \psi \cos \theta & \cos \psi \sin \theta \sin \varphi & \cos \psi \sin \theta \cos \varphi+\sin \psi \sin \varphi \\
\sin \psi \sin \theta & \sin \psi \sin \theta \sin \varphi & \sin \psi \sin \theta \cos \varphi-\cos \psi \sin \varphi \\
-\sin \theta & \cos \theta \sin \varphi & \cos \theta \cos \varphi
\end{array}\right]\left[\begin{array}{l}
x \\
y \\
z
\end{array}\right]
$$

By inverse matrix transformation, we have

$$
\left[\begin{array}{l}
x \\
y \\
z
\end{array}\right]=\left[\begin{array}{ccc}
\cos \psi \cos \theta & \sin \psi \cos \theta & -\sin \theta \\
\cos \psi \sin \theta \sin \varphi-\sin \psi \cos \varphi & \sin \psi \sin \theta \sin \varphi-\cos \psi \cos \varphi & \cos \theta \sin \varphi \\
\cos \psi \sin \theta \cos \varphi+\sin \psi \sin \varphi & \sin \psi \sin \theta \cos \varphi-\cos \psi \sin \varphi & \cos \theta \cos \varphi
\end{array}\right]\left[\begin{array}{l}
\xi \\
\eta \\
\zeta
\end{array}\right]
$$

\subsection{Establishing the Equation of Motion of the Underwater} Robot. Underwater robots are subjected to two types of forces while moving. One is hydrodynamic, i.e., the force exerted by the water on the robot during its navigation. The other type is hydrostatic force, which is the buoyancy and gravity of the underwater robot in the water. Combined with the theory of underwater fluid mechanics and rigid body dynamics, the equation of motion of the underwater robot is researched and established.

In the motion coordinate system of the underwater robot, the force of the underwater robot is $F=[X, Y, Z, K, M, N]^{T}$, and the coordinates of the center of gravity $G$ of the underwater robot can be expressed as $R_{G}=\left(x_{g}, y_{g}, z_{g}\right)^{T}$. According to the theorem of underwater hydrodynamics and velocity synthesis, the velocity $V_{G}$ of the gravity center $G$ of the underwater robot relative to the inertial coordinate system is expressed as

$$
V_{G}=V_{x}+V_{y} \times R_{G} \text {. }
$$

Substituting it into the force formula of the underwater robot, we can get

$$
F=\frac{\mathrm{d} H}{\mathrm{~d} t}=m_{R}\left[V_{x}+V_{y} \times V_{x}+V_{y} \times R_{G}+V_{y}\left(V_{y} \times R_{G}\right)\right] .
$$

Here, $\mathrm{H}$ is the momentum of the underwater robot. By solving the above formulas, three moving mechanics equations of the underwater robot in the motion coordinate system can be obtained.

$$
\begin{aligned}
& X=m_{R}\left(\widehat{u}-v r+w q+z_{g} \widehat{q}+z_{g} p r-x_{g} r^{2}-x_{g} q^{2}\right), \\
& Y=m_{R}\left(\widehat{v}-w r+u r+x_{g} r-z_{g} \widehat{p}+z_{g} q r+x_{g} p q\right), \\
& Z=m_{R}\left(\widehat{w}-u q+v p-x_{g} \widehat{q}+x_{g} p r-z_{g} p^{2}-z_{g} q^{2}\right) .
\end{aligned}
$$

Then, according to the momentum moment theorem, we can get

$$
M_{R}=\frac{\mathrm{d} L}{\mathrm{~d} t}=M_{G}+R_{G} \times F .
$$

Here, $M_{R}$ is the external force moment received by the origin $E$ of the inertial coordinate system, and $M_{G}$ is the external force moment received by the gravity center $G$ of the underwater robot. In the inertial coordinate system, the dynamic moment of the external force on the center of gravity of the underwater robot is expressed as

$$
L_{G}=I_{x g} p \xi+I_{y g} q \eta+I_{z g} r \zeta .
$$

Here, $I_{x g}, I_{y g}$, and $I_{z g}$ are the rotational inertia of the external force on the three coordinate axes of the motion coordinate system of the underwater robot.

According to the moment of inertia theorem, we can get 


$$
\left\{\begin{array}{l}
I_{x}=I_{x g}+m_{R}\left(y_{g}^{2}+z_{g}^{2}\right), \\
I_{y}=I_{y g}+m_{R}\left(z_{g}^{2}+x_{g}^{2}\right), \\
I_{z}=I_{z g}+m_{R}\left(x_{g}^{2}+y_{g}^{2}\right) .
\end{array}\right.
$$

Here, $I_{x}, I_{y}$, and $I_{z}$ are the moment of inertia of the external force on the three coordinate axes of the inertial coordinate system. By solving the above formulas, three rotational mechanics equations of the underwater robot in the motion coordinate system can be obtained.

$$
\begin{aligned}
& K=I_{x} \widehat{p}+I_{x z} \widehat{r}+\left(I_{z x} p+I_{z} r\right) q-I_{y} q r+m_{R}\left(-z_{g} \widehat{v}+z_{g}(w p-u r)\right), \\
& M=I_{y} \widehat{q}+\left(I_{x} p+I_{x z} r\right) r-\left(I_{z x} p+I_{z} r\right) p+m_{R}\left(z_{g} \widehat{u}-x_{g} \widehat{w}+z_{g}(w q-v r)-x_{g}(u q-v p)\right), \\
& N=I_{z x} \widehat{p}+I_{z} \widehat{r}+I_{y} p q-\left(I_{x} p+I_{x z} r\right) q+m_{R}\left(x_{g} \widehat{v}+x_{g}(u r-w p)\right) .
\end{aligned}
$$

Here, $m$ is the mass of the underwater robot, $u, v, w, p, q$, and $r$ are the speed and angular velocity of the six degrees of freedom of the underwater robot, $\widehat{u}, \widehat{v}, \widehat{w}, \widehat{p}, \widehat{q}$, and $\widehat{r}$ are the underwater robot speed and angular velocity of the six degrees of freedom, $x_{g}, y_{g}$, and $z_{g}$ are the coordinates of the center of the underwater robot, $X, Y, Z, K, M$, and $N$ are the moments of the six degrees of freedom, and $I_{x}, I_{y}$, and $I_{z}$ are the underwater robot around moments of inertia of the three axes of the motion coordinate system.

\subsection{Underwater Robot Hydrodynamic Calculation Analysis.} When the underwater robot moves underwater, the hydrodynamic forces it receives can be analyzed into three parts, viscous hydrodynamics, inertial hydrodynamics, and near-wall hydrodynamics. The hydrodynamic model can be expressed as

$$
\tau_{h}=\tau_{i}+\tau_{v}+\tau_{R}
$$

Here, $\tau_{i}$ is inertial hydrodynamic, $\tau_{v}$ is viscous hydrodynamic, and $\tau_{R}$ is near-wall hydrodynamic. The near-wall hydrodynamic force is the additional hydrodynamic force generated by the wall obstruction effect when the underwater robot is moving. The near-wall hydrodynamic force is a function of the speed of the robot and the distance from the wall. In order to complete the inspection of the power plant pool, the underwater robot often moves close to the wall. In this process, the hydrodynamic force of the underwater robot will be more complicated, not only by the longitudinal resistance but also by the hydrodynamic force or moment caused by the wall obstruction effect. Therefore, by obtaining the hydrodynamic model of the underwater robot, this paper studies and establishes the hydrodynamic equation of the underwater robot.

In the underwater longitudinal plane, the force of the underwater robot is decomposed into $F_{c}^{x}$ and $F_{c}^{z}$. Therefore, the force equation of the underwater robot in the underwater longitudinal plane is expressed as

$$
\begin{aligned}
F_{c}^{x} & =-\frac{1}{2} \rho S_{c} V_{c}^{2} \cos ^{2} \alpha_{c}, \\
F_{c}^{z} & =-\frac{1}{4} \rho S_{c} V_{c}^{2} \sin 2 \alpha_{c} .
\end{aligned}
$$

Because the angle of attack is $\alpha_{c}=\alpha_{1}+\delta_{c}$, where $\alpha_{1}$ is approximately 0 , so $\alpha_{c}=\delta_{c}$. The declination angle $\delta_{c}$ has a smaller value, so there are $\cos \delta_{c}=1$ and $\sin 2 \delta_{c} \approx 2 \delta_{c}$. After simplification, the force equation of the underwater robot in the longitudinal plane is

$$
\begin{aligned}
& F_{c}^{x}=-\frac{1}{2} \rho S_{c} V_{c}^{2}, \\
& F_{c}^{z}=\frac{1}{2} \rho S_{c} V_{c}^{2} \delta_{c} .
\end{aligned}
$$

In the underwater lateral plane, the underwater robot moves along the coordinate system, so the force equation of the underwater robot in the underwater lateral plane is expressed as

$$
\begin{aligned}
& F_{f}^{x}=\frac{1}{4} \eta s \rho S_{f} V_{f}^{2} \sin \alpha_{f} \sin 2 \alpha_{f}, \\
& F_{f}^{z}=-\frac{1}{4} \eta s \rho S_{f} V_{f}^{2} \cos \alpha_{f} \sin 2 \alpha_{f} .
\end{aligned}
$$

Among them, $\alpha_{2}$ is relatively small, so there are $\cos \alpha_{2}=$ 1 and $\sin 2 \alpha_{f} \approx 2 \alpha_{f}$. Because the angle of attack $\alpha_{f}=\alpha_{2}+$ $\delta_{f}$ and $\alpha_{2}$ is relatively small, it can be simplified to $\sin \alpha_{2} \approx \alpha_{2}=\left(w+q L_{f}\right) / V$.

After simplification, the force equation of the underwater robot in the lateral plane is

$$
\begin{aligned}
& F_{f}^{x}=\frac{1}{2} \eta s \rho S_{f} V_{f}^{2}\left(\frac{w+q L_{f}}{V}\right)\left(\frac{w+q L_{f}}{V}+\delta_{f}\right), \\
& F_{f}^{z}=-\frac{1}{4} \eta s \rho S_{f} V_{f}^{2}\left(\frac{w+q L_{f}}{V}+\delta_{f}\right) .
\end{aligned}
$$


Then, the hydrodynamic coefficient in the underwater robot model is dimensionless, and the hydrodynamic equation of the robot can be obtained.

$$
\begin{aligned}
X_{h}= & \frac{1}{2} \rho L^{3} X_{u}^{\prime} \widehat{u}+\frac{1}{2} \rho L^{4} X_{q}^{\prime} \widehat{q}+\tau_{R x}\left(V, l_{w}\right), \\
Y_{h}= & \frac{1}{2} \rho L^{3} Y_{v}^{\prime} \widehat{v}+\frac{1}{2} \rho L^{2} Y_{p}^{\prime} \widehat{p}+\tau_{R y}\left(V, l_{w}\right), \\
Z_{h}= & \frac{1}{2} \rho L^{3}\left(Z_{u} \widehat{u}+Z_{w} \widehat{w}\right)+\frac{1}{2} \rho L^{2} Z_{q}^{\prime} u^{2}+\frac{1}{2} \rho L^{2} Z_{w}^{\prime} w u+\tau_{R z}\left(V, l_{w}\right), \\
K_{h}= & \frac{1}{2} \rho L^{4} K_{v}^{\prime} \widehat{v}+\frac{1}{2} \rho L^{5} K_{p}^{\prime} \widehat{p}+\frac{1}{2} \rho L^{3} K_{v}^{\prime} v u+\tau_{R k}\left(V, l_{w}\right), \\
M_{h}= & \frac{1}{2} \rho L^{4} M_{u}^{\prime} \widehat{u}+\frac{1}{2} \rho L^{5} M_{q}^{\prime} \widehat{q}+\frac{1}{2} \rho L^{4} M_{q}^{\prime} q u+\tau_{R m}\left(V, l_{w}\right), \\
N_{h}= & \frac{1}{2} \rho L^{4}\left(N_{v}^{\prime} \widehat{v}+N_{r}^{\prime} r u\right)+\frac{1}{2} \rho L^{5}\left(N_{r}^{\prime} \widehat{r}+N_{r}^{\prime} r|r|\right)+\tau_{R n}\left(V, l_{w}\right) .
\end{aligned}
$$

Here, $L$ is the characteristic length of the underwater robot, and $l_{w}$ is the distance between the underwater robot and the wall.

\section{Dynamic Characteristics Test of the Underwater Robot}

Underwater robotics is a complex system that requires testing and verification of its design, of which underwater motion analysis is an important component. The underwater robot dynamics characteristics are mainly to analyze the hydrodynamic changes caused when the robot moves in the water due to the change of speed magnitude or direction of movement and the reverse effect on the robot. In this paper, the hydrodynamic characteristics of the underwater robot are studied by CFD simulation calculations, and the hydrodynamic test experiments of the underwater robot are carried out in the experimental pool to verify the accuracy of the results of the wide-area hydrodynamic and near-wall hydrodynamic calculations in the underwater robot dynamics model. In this paper, the steady-state and dynamic dynamics of the underwater robot are measured by installing force-sensing sensors on the underwater robot under the action of currents with different flow velocities and then compared with the simulation calculation results to verify the accuracy of the underwater robot dynamics model and numerical simulation. The hydrodynamic test of the underwater robot is shown in Figure 9.

\subsection{Wide-Area Hydrodynamic Test of the Underwater Robot.} In this paper, the hydrodynamic characteristics of the drift angle in the horizontal plane are firstly tested, and the relationship between the wide-area hydrodynamic characteristics of the underwater robot and the drift angle $\beta$ is studied. The velocity of the underwater robot in the process of motion includes longitudinal and lateral velocity components. When the drift angle value of the underwater robot changes, the lateral speed and longitudinal speed of the underwater robot will change, and the bow hydrodynamic force $N$ and lateral hydrodynamic force $Y$ received by the underwater robot will also change accordingly.

In this paper, the longitudinal water velocity $u$ is set to $0.5 \mathrm{~m} / \mathrm{s}$ and $0.8 \mathrm{~m} / \mathrm{s}$ in the test, and the angle of the drift angle $\beta$ is changed at the same time, and the data of the yaw hydrodynamic force $N$ and the lateral hydrodynamic force $Y$ are measured by the force induction sensor. The simulation calculation results are compared with the experimental results, where the red curve is the simulation value and the blue curve is the experimental value, and the corresponding data analysis is performed. The comparison between the experimental and simulation results is shown in Figure 10. Through the comparative analysis of the experimental data, in the horizontal plane, there is an error between the widearea hydrodynamic test results of the underwater robot and the simulation calculation results, but the error range is less than $3.7 \%$, which is within an acceptable range.

The same as above, this paper conducts the test of the hydrodynamic characteristics of the angle of attack in the vertical plane and studies the relationship between the widearea hydrodynamic characteristics of the underwater robot and the angle of attack $\alpha$. When the value of the attack angle $\alpha$ of the underwater robot changes, the vertical power $Z$ and pitch power $M$ are received by the lower robot.

In this paper, the longitudinal water velocity $u$ is set to $0.5 \mathrm{~m} / \mathrm{s}$ and $0.8 \mathrm{~m} / \mathrm{s}$ in the test, and the angle of attack $\alpha$ is changed at the same time, and the data of the vertical force $Z$ and the pitch force $M$ are measured by the force induction sensor. The red curve is the simulation value, and the blue curve is the experimental value. The corresponding data analysis is carried out. The comparison between the experimental and simulation calculation results is shown in Figure 11. Through the comparative analysis of the 


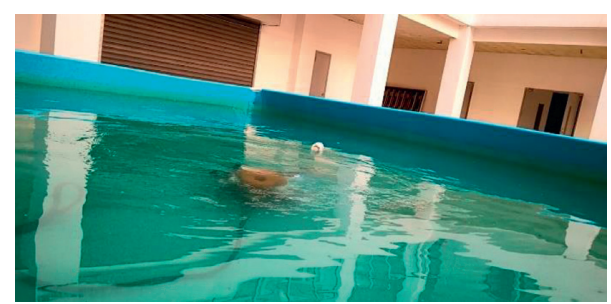

Figure 9: Hydrodynamic test of an underwater robot.

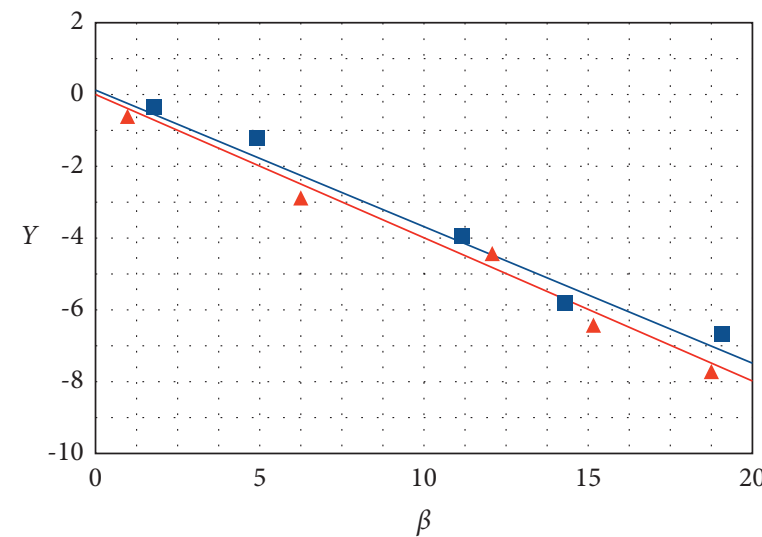

(a)

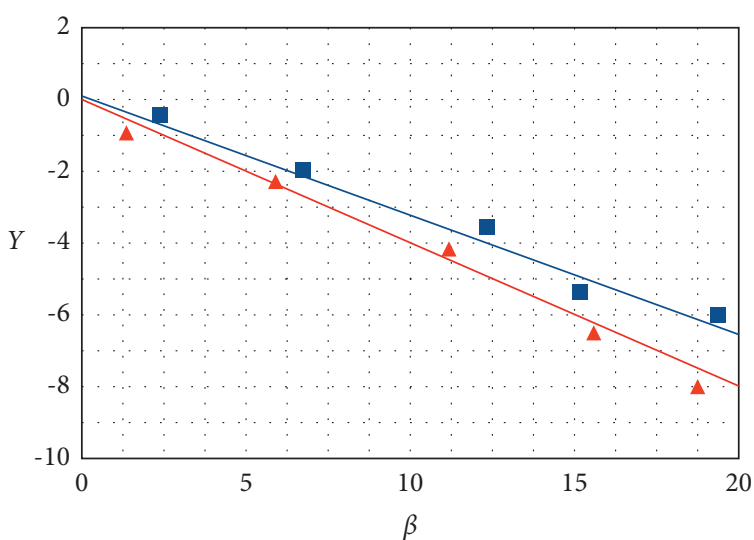

(b)

FiguRE 10: Wide-area hydrodynamic test results and simulation results of underwater robots in the horizontal plane: (a) lateral force test value and simulation value when velocity $=0.5 \mathrm{~m} / \mathrm{s}$ and (b) lateral force test value and simulation value when velocity $=0.8 \mathrm{~m} / \mathrm{s}$.

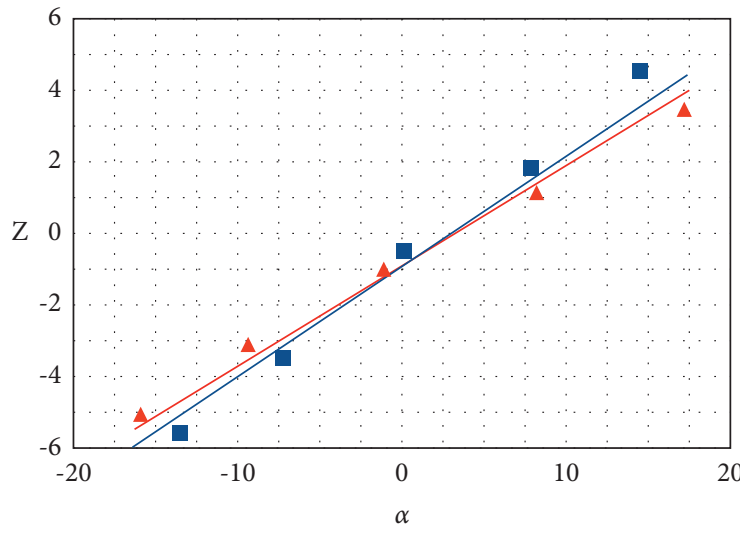

(a)

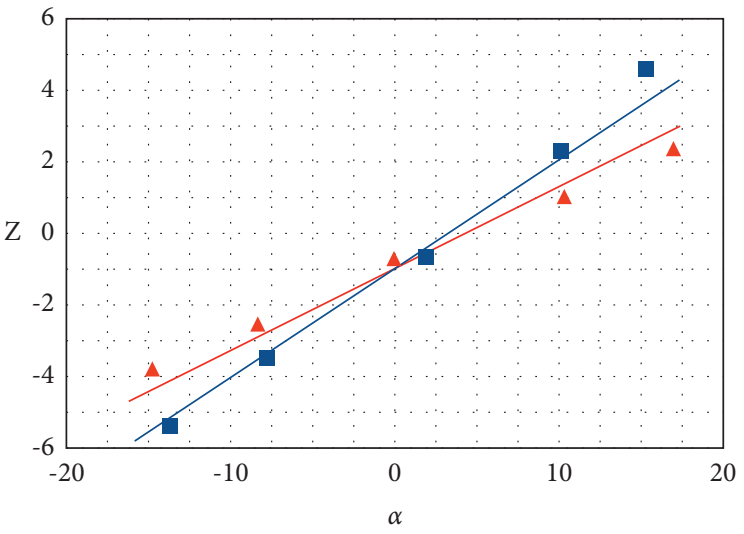

(b)

Figure 11: Wide-area hydrodynamic test results and simulation results of underwater robots in the vertical plane: (a) test value and simulation value of vertical force when velocity $=0.5 \mathrm{~m} / \mathrm{s}$ and $(\mathrm{b})$ test value and simulation value of vertical force when velocity $=0.8 \mathrm{~m} / \mathrm{s}$.

experimental data, there is an error between the results of the wide-area hydrodynamic test of the underwater robot in the vertical plane and the simulation calculation results, but the error range is less than $5.4 \%$, which is within an acceptable range.

\subsection{Underwater Robot Near-Wall Hydrodynamic Testing.} In this paper, the test of the near-wall hydrodynamic characteristics is carried out, the relationship between the near-wall hydrodynamic characteristics of the underwater robot and the distance between the underwater robot and the wall surface is studied, and the underwater robot is tested when the underwater robot moves in the horizontal plane near-wall hydrodynamics. The longitudinal direction of the underwater robot is parallel to the wall of the pool, then the distance between the underwater robot and the near wall is adjusted, and the water velocity is set to $0.5 \mathrm{~m} / \mathrm{s}$ and $0.8 \mathrm{~m} / \mathrm{s}$. The near-wall hydrodynamic changes under different wall distances and water velocity were measured by experiments, and the simulation results were compared with the experimental results. The red curve is the simulation value, and the 


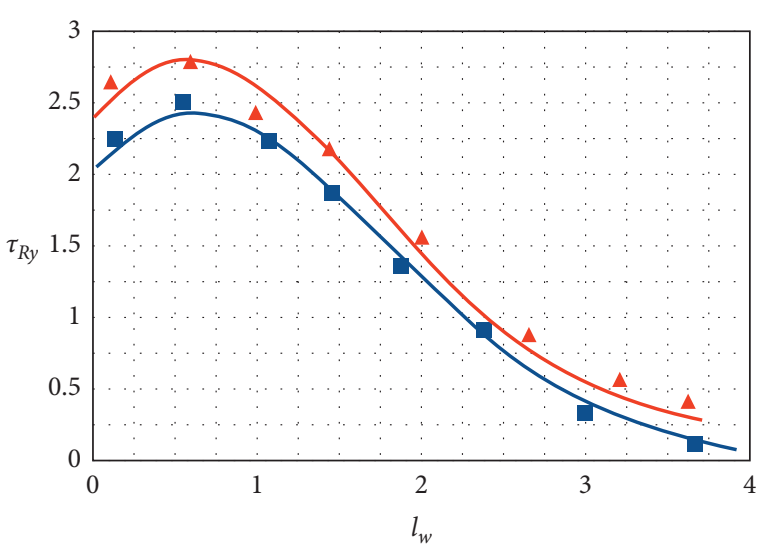

(a)

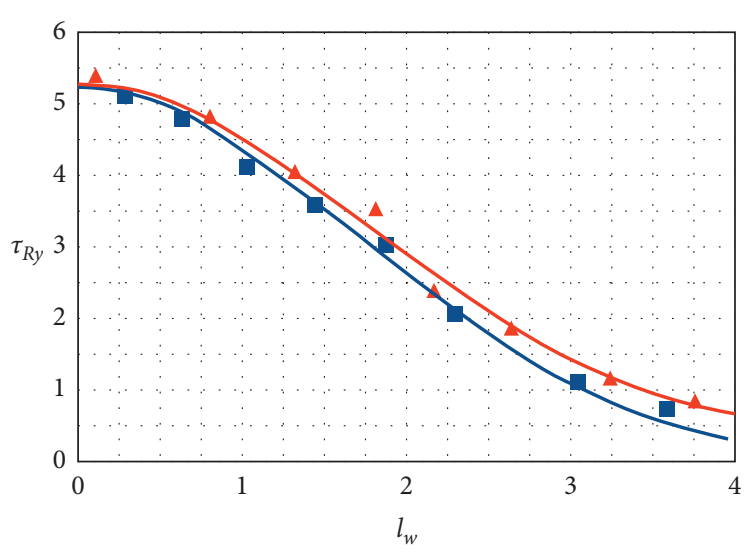

(b)

FIGURE 12: Underwater robot near-wall hydrodynamic test results: (a) test value and simulation value of the near-wall hydrodynamic test when velocity $=0.5 \mathrm{~m} / \mathrm{s}$ and (b) test value and simulation value of the near-wall hydrodynamic test when velocity $=0.8 \mathrm{~m} / \mathrm{s}$.

blue curve is the experimental value. The corresponding data analysis is carried out. The comparison between the experimental and simulation calculation results is shown in Figure 12. Through the comparative analysis of the experimental data, there is a certain error between the results of the hydrodynamic test and the CFD simulation calculation results, but the errors are all below 6.3\%, which is in an acceptable range, which also proves the accuracy of the CFD calculation results.

\section{Conclusion}

In this paper, a new method of underwater robot design is designed to address the problem that underwater robots accumulate errors over time, which affects the positioning and control accuracy of underwater robots. By designing the mechanical structure of the underwater robot, the floating body module, the power unit, the electrical control system, and the software system, the underwater robot is controlled by a combination of software and hardware to accomplish the goals it is intended to achieve. Through experiments, it is proved that the underwater robot designed in this paper is able to complete coordinated movements such as back and forth, rising, floating, and flipping in the water, while adding a mechanical arm at the front of the robot to complete the function of grasping objects in the water, showing the advantages of very good robustness and accuracy. In addition, the robot is equipped with cameras, temperature, humidity, and depth sensors to achieve the monitoring of the water environment. In this paper, the underwater dynamics performance of the underwater robot is studied, the coordinate system and equations of motion are established, hydrodynamic analysis is performed, and the dynamic characteristics of the underwater robot are tested. The comparison between the experimental results and the simulation results shows that the robot meets the requirements of the design indexes in each performance, shows the advantages of good robustness and accuracy, and has high control accuracy and good dynamics characteristics.

\section{Data Availability}

The datasets used to support the findings of this study are available from the corresponding author upon request.

\section{Conflicts of Interest}

The authors declare that there are no conflicts of interest.

\section{Acknowledgments}

This work was supported by the Jiangsu Province Agricultural Science and Technology Independent Innovation Fund Project (CX (21) 1007), open project of Zhejiang Crop Harvest Equipment Technology Key Laboratory (2021KY03 and 2021KY04), Nanjing Institute of Technology Scientific Research Fund Forward-Looking Project (CXY201916), 2021 university-level scientific research fund of Nanjing Institute of Technology (Project no. CKJB202111), and the General Teaching Reform Project of Nanjing Institute of Technology in 2021 (Project no. JXGG2021032).

\section{References}

[1] P. Ma, X. Dai, Z. Guo, C. Wei, and W. Ma, "Detection of thermal pollution from power plants on China's eastern coast using remote sensing data," Stochastic Environmental Research and Risk Assessment, vol. 31, no. 10, pp. 1957-1975, 2017.

[2] R. Hassanzadeh, M. Shams, and M. Darvishyadegari, "An environmentally friendly idea for cooling of water in watercooled chillers in urban areas," International Journal of Advanced Research in Physical Science, vol. 6, no. 1, pp. 7-18, 2019.

[3] L. An, L. Wang, D. Ou et al., "The ecological mechanisms of Acetes blooms as a threat to the security of cooling systems in coastal nuclear power plants," Journal of Coastal Conservation, vol. 25, no. 11, Article ID 55, 2021.

[4] Z. Tang, F. Cheng, X. Jin, L. Sun, R. Bao, and Y. Liu, "An automatic marine-organism monitoring system for the intake water of the nuclear power plant," Annals of Nuclear Energy, vol. 109, no. 11, pp. 208-211, 2017. 
[5] D. Rubio, C. López-Galindo, J. Casanuev, and E. Nebot, "Monitoring and assessment of an industrial antifouling treatment. Seasonal effects and influence of water velocity in an open once-through seawater cooling system," Applied Thermal Engineering, vol. 67, no. 6, pp. 378-387, 2014.

[6] X. Wei, P. Ni, and H. Zhan, "Monitoring cooling water discharge using Lagrangian coherent structures: a case study in Daya Bay, China," Marine Pollution Bulletin, vol. 75, no. 15, pp. 105-113, 2013.

[7] H. Daryanavard and A. Harifi, "UAV path planning for data gathering of IoT nodes: ant colony or simulated annealing optimization," in Proceedings of the 2019 3rd International Conference on Internet of Things and Applications (IoT), Isfahan, Iran, April 2019.

[8] D. Rubio, J. F. Casanueva, and E. Nebot, "Assessment of the antifouling effect of five different treatment strategies on a seawater cooling system," Applied Thermal Engineering, vol. 85, no. 6, pp. 124-134, 2015.

[9] T. Du, J. Hughes, S. Wah, W. Matusik, and D. Rus, "Underwater soft robot modeling and control with differentiable simulation," IEEE Robotics and Automation Letters, vol. 6, no. 3, pp. 4994-5001, 2021.

[10] E. K. Petavratzis, C. K. Volos, I. N. Stouboulos, I. M. Kyprianidis, H. E. Nistazakis, and G. S. Tombras, "Robot's path planning based on emulated finite resistive grids," in Proceedings of the 2018 7th International Conference on Modern Circuits and Systems Technologies (MOCAST), Thessaloniki, Greece, May 2018.

[11] S.-M. Lee, M.-I. Roh, K.-S. Kim, H. Jung, and J. J. Park, "Method for a simultaneous determination of the path and the speed for ship route planning problems," Ocean Engineering, vol. 157, pp. 301-312, 2018.

[12] D. Wei, L. Yan, C. Huang et al., "Dynamic magnetic induction wireless communications for autonomous-underwater-vehicle-assisted underwater IoT," IEEE Internet of Things Journal, vol. 7, no. 10, pp. 9834-9845, 2020.

[13] R. Bogue, "Underwater robots: a review of technologies and applications," Industrial Robot: International Journal, vol. 42, no. 3, pp. 186-191, 2015.

[14] J. Zheng, C. Huntrakul, X. Guo, C. Wang, and G. Xie, "Electric sense based pose estimation and localization for small underwater robots," IEEE Robotics and Automation Letters, vol. 7, no. 2, pp. 2835-2842, 2022.

[15] S. Sedighi, D. Nguyen, and K. Kuhnert, "Guided hybrid A-star path planning algorithm for valet parking applications," in Proceedings of the 2019 5th International Conference on Control Automation and Robotics (ICCAR), Beijing, China, April 2019.

[16] B. K. Patle, G. Babu L, A. Pandey, D. R. K. Parhi, and A. Jagadeesh, "A review: on path planning strategies for navigation of mobile robot," Defence Technology, vol. 15, no. 4, pp. 582-606, 2019.

[17] I. Moskvin, R. Lavrenov, E. Magid, and M. Svinin, "Modelling a crawler robot using wheels as pseudo-tracks: model complexity vs. performance," in Proceedings of the 2020 IEEE 7th International Conference on Industrial Engineering and Applications (ICIEA), Beijing, China, April 2020.

[18] I. Ullah, J. Chen, X. Su, C. Esposito, and C. Choi, "Localization and detection of targets in underwater wireless sensor using distance and angle based algorithms," IEEE Access, vol. 7, pp. 45693-45704, 2019.

[19] O. Guarnizo and I. Pineda, "Three dimensional adaptive path planning simulation based on ant colony optimization algorithm," in Proceedings of the 2019 IEEE Latin American
Conference on Computational Intelligence (LA-CCI), Guayaquil, Ecuador, November 2019.

[20] M. E. Yuksel, "Agent-based evacuation modeling with multiple exits using neuroEvolution of augmenting topologies," Advanced Engineering Informatics, vol. 35, no. 11, pp. 30-55, 2018.

[21] A. I. Panov, K. S. Yakovlev, and R. Suvorov, "Grid path planning with deep reinforcement learning: preliminary results," Procedia Computer Science, vol. 123, pp. 347-353, 2018.

[22] F. Qu, S. Wang, Z. Wu, and Z. Liu, "A survey of ranging algorithms and localization schemes in underwater acoustic sensor network," China Communications, vol. 13, no. 3, pp. 66-81, 2016.

[23] M. Ishida, D. Drotman, B. Shih, M. Hermes, M. Luhar, and M. T. Tolley, "Morphing structure for changing hydrodynamic characteristics of a soft underwater walking robot," IEEE Robotics and Automation Letters, vol. 4, no. 4, pp. 4163-4169, 2019.

[24] M. Calisti, F. Corucci, A. Arienti, and C. Laschi, "Dynamics of underwater legged locomotion: modeling and experiments on an octopus-inspired robot," Bioinspiration \& Biomimetics, vol. 10, no. 4, Article ID 046012, 2015. 